\title{
Efficient transformed ratio-type estimator using single auxiliary information
}

\author{
Sana Amjad ${ }^{1}$, Muhammad Ismail ${ }^{2, *}$ \\ ${ }^{1}$ Dept. of Statistics, University of the Punjab Lahore, Pakistan \\ ${ }^{2}$ Dept. of Statistics, COMSATS University Islamabad, Lahore Campus Lahore, Pakistan \\ *Corresponding author:drismail39@gmail.com
}

\begin{abstract}
This paper provides an efficient transformed ratio-type estimator to estimate the study variable's population variance by utilizing information of a single auxiliary variable under simple random sampling without replacement. The bias and mean squared error of the proposed estimator are derived up-to 1st order approximation. In addition to this, the efficiency comparison of the proposed estimator has been done with traditional ratio-type variance estimator and some other widely used modified ratio-type variance estimators by taking real-life data. A simulation study has also been carried out to see the performance of the proposed estimator. It is worth noticing that our proposed estimator performs better than the competing estimators in real-life data applications as the mean squared error and root mean squared error of our proposed estimator are smaller than the competing estimators. Hence, our proposed estimator is better than existing variance estimators.
\end{abstract}

Keywords: Auxiliary information; bias; mean squared error; simple random sampling; study variable.

\section{Introduction}

The estimation of the population variance has significance in different life areas, e.g., industries, agriculture, and medical sciences. It is conventional that the population variance is unknown and has to be estimated using given data. In this paper, an attempt has been made to estimate this unknown characteristic reasonably and efficiently. The principle aim of this paper is to propose a method that can be used to estimate dispersion efficiently. Various estimators are available in the literature to estimate variation, including ratio, regression, transformed regression, and ratiotype variance estimators. Some of the authors used the above techniques in randomized response models (RTT) like Singh \& Suman, 2019.
Consider a finite population of size $\mathrm{N}$ from which a sample of size $n$ is to be drawn under simple random sampling (SRSWOR). Suppose $\mathrm{y}$ denotes the study variable, and $\mathrm{x}$ is auxiliary information about which some information is available. Some necessary notations to be used in the paper are given as

$\bar{Y}, S_{Y}^{2}$ : Population mean and variance of $Y$

$\bar{X}, S_{X}^{2}$ : Population mean and variance of $X$

The sample counterparts of means and variances will be denoted by small case roman letters. $\rho:$ The population correlation coefficient, $r$ : The sample correlation coefficient, and $S_{x y}$ : Covariance between $\mathrm{X}$ and $\mathrm{Y}$. The coefficient of variations for $Y$ and 
$X$ will be denoted by $C_{y}$ and $C_{x}$. Also $\beta_{2}^{\prime}(y)=\mu_{40} / \mu_{20}^{2}$ and $\beta_{2}^{\prime}(x)=\mu_{04} / \mu_{02}^{2}$ be the coefficient of kurtosis for $Y$ and $X$, respectively where,

$$
\mu_{s t}=\frac{1}{N} \sum_{i=1}^{n}\left(Y_{i}-\bar{Y}\right)^{s}\left(X_{i}-\bar{X}\right)^{t}
$$

is $(s, t)$ the central order moment. Some additional notations are $\lambda^{\prime}=n^{-1}$, $\lambda=(N n)^{-1}(N-n)$ and $a=\mu_{22} / \mu_{20} \mu_{02}$.

Some important expectations given by Yadav et al., 2013, which are useful in computing mean square error of an estimator, are given as

$$
\begin{aligned}
& E\left(e_{0}\right)=0, \quad E\left(e_{1}\right)=0, \quad E\left(e_{0}^{2}\right)=\lambda \beta_{2}^{\wedge}(y) \\
& E\left(e_{1}^{2}\right)=\lambda \beta_{2}^{\wedge}(x) \quad, \quad E\left(e_{0} e_{1}\right)=\lambda a^{\prime} \\
& \beta_{2}^{\wedge}(y)=\beta_{2}^{\prime}(y)-1, \quad \beta_{2}^{\wedge}(x)=\beta_{2}^{\prime}(x)-1 \\
& a^{\prime}=a-1 \quad, \quad \mu^{\prime}=\frac{a^{\prime}}{\beta_{2}^{\wedge}(x)}
\end{aligned}
$$

For estimation of the population variance, Isaki, 1983, proposed a ratio-type estimator by using one auxiliary information (x). Some other references are Upadhyaya \& Singh, 1999; Ahmed et al., 2000; Kadilar \& Cingi, 2006; Subramani \& Kumarapandiyan, 2012a, b; Subramani \& Kumarapandiyan, 2013; Chami et al., 2012; Singh \& Solanki, 2013a, b; Yadav \& Kadilar, 2014; Ismail et al., 2018. and Akhlaq et al., 2019. Few biased variance estimators, as well as their bias and mean squared errors, are given below:

Isaki, 1983, ratio-type estimator

$$
t_{I s}=s_{y}^{2}\left(\frac{S_{x}^{2}}{s_{x}^{2}}\right)
$$

With bias and mean squared error given as
$\operatorname{BIAS}\left(t_{I s}\right) \cong \lambda S_{y}^{2}\left\{\beta_{2}^{\wedge}(x)-a^{\prime}\right\}$

$\operatorname{MSE}\left(t_{I s}\right) \cong \lambda S_{y}^{4}\left\{\beta_{2}^{\wedge}(y)+\beta_{2}^{\wedge}(x)-2 a^{\prime}\right\}$.

Upadhyaya \& Singh, 1999, suggested following ratio-type estimator

$t_{u p s i}=s_{y}^{2}\left[\frac{S_{x}^{2}+\beta_{2}^{\prime}(x)}{s_{x}^{2}+\beta_{2}^{\prime}(x)}\right]$

With bias and mean squared error given as

$$
\begin{aligned}
& \operatorname{BIAS}\left(t_{\text {upsi }}\right) \cong\left[\begin{array}{l}
\lambda S_{y}^{2} \beta_{2}^{\wedge}(x) \frac{S_{x}^{2}}{S_{x}^{2}+\beta_{2}^{\prime}(x)} \\
\left(\frac{S_{x}^{2}}{S_{x}^{2}+\beta_{2}^{\prime}(x)}-\mu^{\prime}\right)
\end{array}\right] \\
& \operatorname{MSE}\left(t_{\text {upsi }}\right) \cong \lambda S_{y}^{4}\left[\begin{array}{l}
\beta_{2}^{\wedge}(y)+\beta_{2}^{\wedge}(x) \frac{S_{x}^{2}}{S_{x}^{2}+\beta_{2}^{\prime}(x)} \\
\left(\frac{S_{x}^{2}}{S_{x}^{2}+\beta_{2}^{\prime}(x)}-2 \mu^{\prime}\right)
\end{array}\right]
\end{aligned}
$$

Kadilar \& Cingi, 2006, proposed following four modified ratio type estimators

$$
\begin{aligned}
& t_{K C 1}=s_{y}^{2}\left[\frac{S_{x}^{2}-\beta_{2}^{\prime}(x)}{s_{x}^{2}-\beta_{2}^{\prime}(x)}\right] \\
& t_{K C 2}=s_{y}^{2}\left[\frac{C_{x} S_{x}^{2}-\beta_{2}^{\prime}(x)}{C_{x} s_{x}^{2}-\beta_{2}^{\prime}(x)}\right] \\
& t_{K C 3}=s_{y}^{2}\left[\frac{S_{x}^{2}-C_{x}}{s_{x}^{2}-C_{x}}\right] \\
& t_{k c 4}=s_{y}^{2}\left[\frac{\beta_{2}^{\prime}(x) S_{x}^{2}-C_{x}}{\beta_{2}^{\prime}(x) s_{x}^{2}-C_{x}}\right]
\end{aligned}
$$

with biases and mean squared errors given as

$$
\operatorname{BIAS}\left(t_{K C i}\right) \cong \lambda S_{y}^{2} \beta_{2}^{\wedge}(x) B_{i}\left(B_{i}-\mu^{\prime}\right)
$$


$\operatorname{MSE}\left(t_{K C i}\right) \cong \lambda S_{y}^{4}\left[\beta_{2}^{\wedge}(y)+\beta_{2}^{\wedge}(x) B_{i}\left(B_{i}-2 \mu^{\prime}\right)\right]$

$i=1,2,3,4$

$B_{1}=\frac{S_{x}^{2}}{S_{x}^{2}-\beta_{2}^{\prime}(x)} \quad, \quad B_{2}=\frac{C_{x} S_{x}^{2}}{C_{x} S_{x}^{2}-\beta_{2}^{\prime}(x)}$

$B_{3}=\frac{S_{x}^{2}}{S_{x}^{2}-C_{x}} \quad, \quad B_{4}=\frac{\beta_{2}^{\prime}(x) S_{x}^{2}}{\beta_{2}^{\prime}(x) S_{x}^{2}-C_{x}}$

Chami et al., 2012, estimator

$t_{c h}=\bar{y}\left[\alpha\left\{\frac{(1-\beta) \bar{x}+\beta \bar{X}}{\beta \bar{x}+(1-\beta) \bar{X}}\right\}+(1-\alpha)\left\{\frac{\beta \bar{x}+(1-\beta) \bar{X}}{(1-\beta) \bar{x}+\beta \bar{X}}\right\}\right]$ (7)

with bias and mean squared error

$\operatorname{BIAS}\left(t_{c h}\right)=\frac{1-f}{n}(1-2 \beta)[1-\alpha-\beta-(1-2 \alpha) C] C_{x}^{2} \bar{Y}$

$\operatorname{MSE}\left(t_{c h}\right)=\frac{1-f}{n} S_{Y}^{2}\left(1-\rho^{2}\right)$

Ismail et al., 2018, estimator

$t_{R P G}=\left(s_{y}^{2}+a\right)\left[\begin{array}{l}\lambda\left(\frac{S_{g}^{2}}{s_{g}^{2}}\right)^{\tau}+(1-\lambda-\omega) \\ \left(\frac{s_{g}^{2}}{S_{g}^{2}}\right)^{\chi}+\omega\left(\frac{S_{n}^{2}}{s_{n}^{2}}\right)^{\vartheta}\end{array}\right]-a$

with bias and mean squared error

$\operatorname{Bias}\left(t_{R P G}\right) \cong \frac{1}{2} \lambda S_{y}^{2} \vartheta_{0}\left[\begin{array}{l}\beta_{2}^{\wedge}(x) h_{1}^{2}\left\{\lambda \tau^{2}+(1-\lambda-\omega) \chi^{2}\right\}+ \\ \beta_{2}^{\wedge}(x)\{\lambda \tau-(1-\lambda-\omega) \chi\} h_{1}\left\{h_{1}-2 \mu^{\prime}\right\}+ \\ \beta_{2}^{\wedge}(z) \omega \theta^{2} h_{2}^{2}+\beta_{2}^{\wedge}(z) \omega \theta h_{2}\left(h_{2}-2 v^{\prime}\right)\end{array}\right]$

$\operatorname{MSE}\left(t_{R P G}\right) \cong \lambda S_{y}^{4}\left[\begin{array}{l}\beta_{2}^{\wedge}(y)+\beta_{2}^{\wedge}(x) N_{1}\left(N_{1}-2 \mu^{\prime}\right)+ \\ \beta_{2}^{\wedge}(z) N_{2}\left(N_{2}-2 v^{\prime}\right)+2 N_{1} N_{2} c^{\prime}\end{array}\right]$

where

$N_{1}=\left(\beta_{2}^{\wedge}(z) a^{\prime}-b^{\prime} c^{\prime}\right) / E$

$N_{2}=\left(\beta_{2}^{\wedge}(x) b^{\prime}-a^{\prime} c^{\prime}\right) / E$

and

$E=\beta_{2}^{\wedge}(x) \beta_{2}^{\wedge}(z)-c^{\prime 2}$
2. Proposed efficient transformed ratio type estimator using single auxiliary information

By taking motivation from Chami et al., 2012, we have suggested a new estimator based on the single auxiliary variate information.

$$
t_{s a}=s_{y}^{2}\left[\left\{\frac{S_{x}^{2}-\beta_{x y}\left(S_{x}^{2}-s_{x}^{2}\right)}{s_{x}^{2}+\beta_{x y}\left(S_{x}^{2}-s_{x}^{2}\right)}\right\}^{\alpha}\left\{\frac{S_{x}^{2}+\left(S_{x}^{2}-s_{x}^{2}\right)}{s_{x}^{2}-\left(S_{x}^{2}-s_{x}^{2}\right)}\right\}^{1-\alpha}\right]
$$

We aim to derive the value of constant $\alpha$ such that the mean squared error (MSE) and root mean squared error (RMSE) of the proposed estimator $t_{s a}$ are minimum. In order to study the large sample properties of the proposed estimator $t_{s a}$, we define $s_{y}^{2}=S_{y}^{2}\left(1+e_{0}\right)$ and $s_{x}^{2}=S_{x}^{2}\left(1+e_{1}\right)$.

Now replacing $s_{y}^{2}$ and $s_{x}^{2}$ in equation (9), we have

$$
t_{s a}=S_{y}^{2}\left(1+e_{0}\right)\left[\begin{array}{l}
\left\{\frac{S_{x}^{2}-\beta_{x y}\left(S_{x}^{2}-S_{x}^{2}\left(1+e_{1}\right)\right)}{S_{x}^{2}\left(1+e_{1}\right)+\beta_{x y}\left(S_{x}^{2}-S_{x}^{2}\left(1+e_{1}\right)\right)}\right\}^{\alpha} \\
\left\{\frac{S_{x}^{2}+\left(S_{x}^{2}-S_{x}^{2}\left(1+e_{1}\right)\right)}{S_{x}^{2}\left(1+e_{1}\right)-\left(S_{x}^{2}-S_{x}^{2}\left(1+e_{1}\right)\right)}\right\}^{1-\alpha}
\end{array}\right]
$$

By retaining powers up to $e_{1}^{2}$ we get

$$
t_{s a}-S_{y}^{2} \cong S_{y}^{2}\left[\begin{array}{l}
e_{0}-3 e_{1}-3 e_{0} e_{1}+2 \alpha e_{1}+ \\
2 \alpha e_{0} e_{1}+2 e_{1}^{2}-\alpha e_{1}^{2}-\alpha^{2} e_{1}^{2}+ \\
2 \alpha \beta_{x y} e_{1}+2 \alpha \beta_{x y} e_{0} e_{1}-6 \alpha e_{1}^{2} \beta_{x y}+ \\
6 \alpha^{2} e_{1}^{2} \beta_{x y}-\alpha^{2} \beta_{x y} e_{1}^{2}+\alpha^{2} e_{1}^{2} \beta_{x y}^{2}
\end{array}\right]
$$

Bias of new estimator $t_{s a}$ up-to 1st-degree approximation is

$$
B I A S\left(t_{s a}\right) \cong \lambda S_{y}^{2}\left[\begin{array}{l}
-3 a^{\prime}+2 \alpha a^{\prime}+2 \beta_{2}^{\wedge}(x)-\alpha \beta_{2}^{\wedge}(x)- \\
\alpha^{2} \beta_{2}^{\wedge}(x)+2 \alpha \beta_{x y} a^{\prime}-6 \alpha \beta_{x y} \beta_{2}^{\wedge}(x)+ \\
5 \alpha^{2} \beta_{x y} \beta_{2}^{\wedge}(x)+\alpha^{2} \beta_{x y}^{2} \beta_{2}^{\wedge}(x)
\end{array}\right]
$$


The mean squared error (MSE) of the new estimator $t_{s a}$ is

$$
M S E\left(t_{s a}\right) \cong \lambda S_{y}^{4}\left[\begin{array}{l}
\beta_{2}^{\wedge}(y)+9 \beta_{2}^{\wedge}(x)+4 \alpha^{2} \beta_{2}^{\wedge}(x)+ \\
4 \alpha^{2} \beta_{x y}^{2} \beta_{2}^{\wedge}(x)-6 a^{\prime}+4 \alpha a^{\prime}+4 \alpha \beta_{x y} a^{\prime}- \\
12 \alpha \beta_{2}^{\wedge}(x)-12 \alpha \beta_{x y} \beta_{2}^{\wedge}(x)+8 \alpha^{2} \beta_{x y} \beta_{2}^{\wedge}(x)
\end{array}\right]
$$

Where the optimal value of $\alpha$ is attained by reducing the above equation and is given as $\alpha=\left[\frac{\left(-a^{\prime}+3 \beta_{2}^{\wedge}(x)\right)\left(1+\beta_{x y}\right)}{2 \beta_{2}^{\wedge}(x)\left(1+\beta_{x y}^{2}+2 \beta_{x y}\right)}\right]$

By utilizing the optimal value of $\alpha$ the minimum mean squared error of proposed estimator is

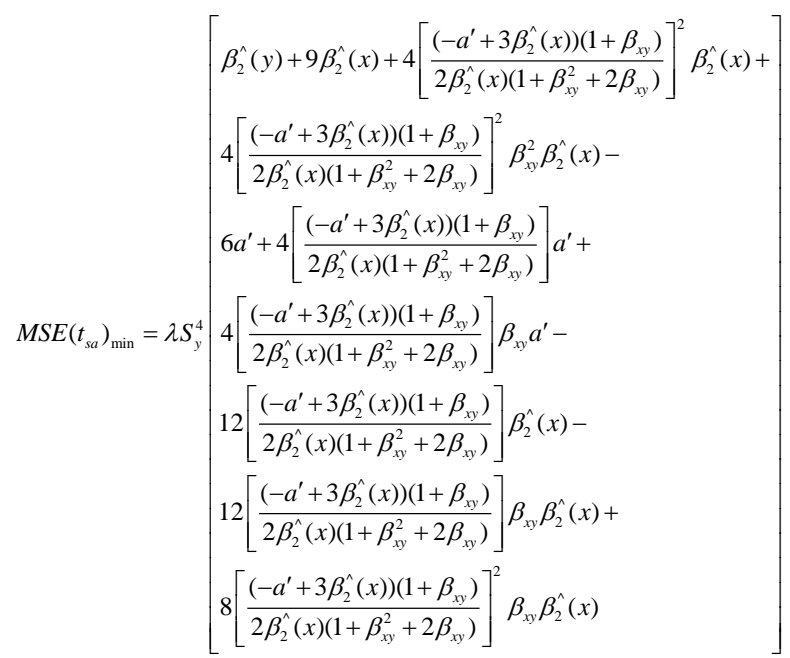

\section{Special case of new proposed estimator of population variance}

If we put $\alpha=1$ and $\beta_{x y}=0$, the proposed estimator reduces to Isaki, 1983 ratio estimator.

\section{Results}

This section provides twofold studies. Firstly, an empirical analysis is carried out by considering two real populations; later on, a simulation study is conducted to prove the suitability of the new estimator $t_{s a}$.

\subsection{Empirical analysis}

To carry out the empirical analysis, 2 data sets are occupied from Basic Econometrics (2004) by Gujarati and Sangeetha.

Data 01: Data is collected about 81cars where variable $\mathrm{y}$ is the average no of covered miles, and variable $\mathrm{x}$ is engine horsepower. The data summary measures are given in Table 1, whereas the computed values of mean squared error and root mean squared errors are given in Table 2.

Table 2 compares the proposed estimator $t_{s a}$ over traditional ratio-type variance estimator and widely used modified ratio-type variance estimators. It can be seen that as the sample size (n) increases, the mean squared error (MSE) and root mean squared error (RMSE) decreases. The smaller the value of mean squared error (MSE) and root mean squared error (RMSE) indicates the more extensive the percentage relative efficiency (PRE). Table 2 shows that the proposed estimator $t_{s a}$ has less MSE, RMSE, and larger percentage relative efficiency (PRE) overall existing estimators

Table 1. Data description.

\begin{tabular}{c|ccccc}
\hline$C_{y}$ & $C_{x}$ & $S_{y}^{2}$ & $S_{x}^{2}$ & $\beta_{2}^{\prime}(y)$ \\
\hline 0.2971934 & 0.4862001 & 101.1113 & 3261.952 & 3.591309 \\
$\beta_{2}^{\prime}(x)$ & $a$ & $\rho_{x y}$ & $\mathrm{n}$ & \\
5.707267 & 2.230698 & -0.7944504 & 10,20 & \\
\hline
\end{tabular}


Table 2. Mean squared error (MSE), and root mean squared error (RMSE), and percentage relative efficiency (PRE) of different estimators.

\begin{tabular}{c|ccc|c|c|c}
\hline \multirow{2}{*}{ Estimators } & \multicolumn{2}{c|}{ MSE } & \multicolumn{2}{c|}{ RMSE } & \multicolumn{2}{c}{ PRE } \\
\cline { 2 - 7 } & $\mathbf{n = 1 0}$ & $\mathbf{n = 2 0}$ & $\mathbf{n = 1 0}$ & $\mathbf{n = 2 0}$ & $\mathbf{n = 1 0}$ & $\mathbf{n = 2 0}$ \\
\hline$t_{0}$ & 2322.159 & 997.547 & 48.18878 & 31.58397 & 100 & 100 \\
$t_{\text {Is }}$ & 4334.759 & 1862.115 & 65.83888 & 43.15223 & 53.57065 & 53.57065 \\
$t_{\text {upsi }}$ & 4323.889 & 1857.445 & 65.75628 & 43.09809 & 53.70533 & 53.70533 \\
$t_{\text {KC1 }}$ & 4345.693 & 1866.812 & 65.92187 & 43.20662 & 53.43587 & 53.43587 \\
$t_{\text {KC2 }}$ & 4357.317 & 1871.805 & 66.00998 & 43.26437 & 53.29332 & 53.29332 \\
$t_{K C 3}$ & 4335.688 & 1862.514 & 65.84594 & 43.15685 & 53.55918 & 53.55918 \\
$t_{K C 4}$ & 4334.921 & 1862.185 & 65.84012 & 43.15304 & 53.56865 & 53.56865 \\
$t_{\text {suku } 1}$ & 4153.154 & 1784.101 & 64.44497 & 42.23862 & 55.91314 & 55.91314 \\
$t_{\text {suku } 2}$ & 4186.259 & 1798.323 & 64.70131 & 42.40663 & 55.47098 & 55.47098 \\
$t_{\text {suku } 3}$ & 4085.482 & 1755.031 & 63.91777 & 41.89309 & 56.83929 & 56.83929 \\
$t_{\text {suku4 } 4}$ & 4225.391 & 1815.133 & 65.00301 & 42.60438 & 54.95725 & 54.95725 \\
$t_{\text {suku } 5}$ & 4279.252 & 1838.27 & 65.41599 & 42.87505 & 54.26553 & 54.26553 \\
$t_{\text {suku } 6}$ & 4135.128 & 1776.358 & 64.30496 & 42.14686 & 56.15688 & 56.15688 \\
$t_{\text {suku } 7}$ & 3980.021 & 1709.727 & 63.08741 & 41.34885 & 58.34539 & 58.34539 \\
$t_{\text {sa }}$ & 2033.817 & 873.6821 & 45.09786 & 29.55811 & 114.1773 & 114.1773 \\
\hline
\end{tabular}

Data 02: Data is collected about literacy rate of 64 countries where $\mathrm{y}$ : no of children died under age five in a $\quad \mathrm{x}$ : total fertility rate year per 1000 live births

Table 3. Data description.

\begin{tabular}{l|l|l|l|l}
\hline$C_{y}$ & $C_{x}$ & $S_{y}^{2}$ & $\beta_{2}^{\prime}(y)$ & $\beta_{2}^{\prime}(x)$ \\
\hline 0.5369475 & 0.271906 & 5772.667 & 2.378336 & 2.816908 \\
$a$ & $\rho_{x y}$ & $S_{x}^{2}$ & $\mathrm{n}$ & \\
1.481965 & 0.6711349 & 2.27706 & 8,16 & \\
\hline
\end{tabular}


Table 4. Mean squared error (MSE), and root mean squared error (RMSE), and percentage relative efficiency (PRE) of different estimators.

\begin{tabular}{|c|c|c|c|c|c|c|}
\hline \multirow[t]{2}{*}{ Estimators } & \multicolumn{2}{|c|}{ MSE } & \multicolumn{2}{|c|}{ RMSE } & \multicolumn{2}{|c|}{ PRE } \\
\hline & $n=8$ & $n=16$ & $n=8$ & $n=16$ & $\mathrm{n}=\mathbf{8}$ & $n=16$ \\
\hline$t_{0}$ & 5023729 & 2153027 & 2241.368 & 1467.32 & 100 & 100 \\
\hline$t_{I s}$ & 8132649 & 3485421 & 2851.78 & 1866.928 & 61.77235 & 61.77235 \\
\hline$t_{u p s i}$ & 4776488 & 2047066 & 2185.518 & 1430.757 & 105.17620 & 105.17620 \\
\hline$t_{K C 1}$ & 137660302 & 58997272 & 11732.87 & 7680.968 & 3.64936 & 3.64936 \\
\hline$t_{K C 2}$ & 6539055 & 2802452 & 2557.158 & 1674.053 & 76.82652 & 76.82652 \\
\hline$t_{K C 3}$ & 9573998 & 4103142 & 3094.188 & 2025.621 & 52.47263 & 52.47263 \\
\hline$t_{K C 4}$ & 8576397 & 3675599 & 2928.549 & 1917.185 & 58.57621 & 58.57621 \\
\hline$t_{s u k u 1}$ & 4558229 & 1953527 & 2135.001 & 1397.686 & 110.21229 & 110.21229 \\
\hline$t_{\text {suku } 2}$ & 4592052 & 1968022 & 2142.907 & 1402.862 & 109.40052 & 109.40052 \\
\hline$t_{\text {suku3 }}$ & 4558361 & 1953583 & 2135.032 & 1397.706 & 110.20910 & 110.20910 \\
\hline$t_{\text {suku } 4}$ & 4966698 & 2128585 & 2228.609 & 1458.967 & 110.11482 & 110.11482 \\
\hline$t_{\text {suku5 }}$ & 5690191 & 2438653 & 2385.412 & 1561.619 & 88.28752 & 88.28752 \\
\hline$t_{\text {suku6 }}$ & 4562076 & 1955175 & 2135.902 & 1398.276 & 110.11936 & 110.11936 \\
\hline$t_{\text {suku7 }}$ & 4754320 & 2037566 & 2180.44 & 1427.433 & 105.66661 & 105.66661 \\
\hline$t_{s a}$ & 4557748 & 1953321 & 2134.888 & 1397.613 & 110.2239 & 110.2239 \\
\hline
\end{tabular}

Table 4 represents the suitability of the proposed estimator $t_{s a}$ over traditional ratiotype variance estimator and widely used modified ratio-type variance estimators. It is evident from table 4 that as sample size (n) increases, the mean squared error (MSE) and root mean squared error (RMSE) decreases. The smaller the value of mean squared error (MSE) and root mean squared error (RMSE) indicates the more extensive the percentage relative efficiency (PRE). In table 4 , the proposed estimator $t_{s a}$ shows less MSE, RMSE, and larger percentage relative efficiency (PRE) overall existing estimators.

\subsection{Simulation study results}

In this section, a simulation study is done to see the performance of the suggested estimator $t_{s a}$. A simulation study is carried out by using a multivariate normal distribution, and we generated a population of size 10,000 . This process is repeated 1000 times, and then we computed MSE, RMSE, and PRE of the proposed and existing estimators.

Table 5 represents that the new suggested estimator $t_{s a}$ is showing less (MSE) and (RMSE) as well as larger percentage relative efficiency (PRE) among all existing estimators. 
Table 5. Mean squared error (MSE), and root mean squared error (RMSE), and percentage relative efficiency (PRE) at different values of correlation coefficients.

\begin{tabular}{|c|c|c|c|}
\hline \multirow[t]{2}{*}{ Estimators } & MSE & RMSE & PRE \\
\hline & $r=0.88$ & $r=0.88$ & $\mathrm{r}=0.88$ \\
\hline$t_{0}$ & 0.0001987291 & 0.01441634 & 100 \\
\hline$t_{I s}$ & $8.829262 \mathrm{e}-05$ & 0.009461709 & 216.9821 \\
\hline$t_{u p s i}$ & 0.0001345604 & 0.011834 & 147.9304 \\
\hline$t_{K C 1}$ & 0.0003945465 & 0.02020014 & 49.55449 \\
\hline$t_{K C 2}$ & $8.561994 \mathrm{e}-05$ & 0.009442035 & 221.7021 \\
\hline$t_{K C 3}$ & 0.0001954534 & 0.01440238 & 101.1279 \\
\hline$t_{K C 4}$ & 0.0001889825 & 0.01437263 & 103.3942 \\
\hline$t_{s u k u 1}$ & $9.075502 \mathrm{e}-05$ & 0.009523128 & 216.2621 \\
\hline$t_{\text {suku } 2}$ & 0.001098177 & 0.03672747 & 15.1962 \\
\hline$t_{\text {suku } 3}$ & $8.437891 \mathrm{e}-05$ & 0.009226042 & 231.697 \\
\hline$t_{\text {suku } 4}$ & 0.0001024148 & 0.01024249 & 191.5811 \\
\hline$t_{\text {suku } 5}$ & $8.440008 \mathrm{e}-05$ & 0.009237856 & 230.711 \\
\hline$t_{\text {suku } 6}$ & $8.836804 \mathrm{e}-05$ & 0.00950407 & 213.4412 \\
\hline$t_{\text {suku } 7}$ & $8.826834 \mathrm{e}-05$ & 0.009461637 & 216.9873 \\
\hline$t_{s a}$ & $7.817046 \mathrm{e}-05$ & 0.0088582 & 247.0853 \\
\hline
\end{tabular}


Efficiency comparison with respect to correlation coefficients

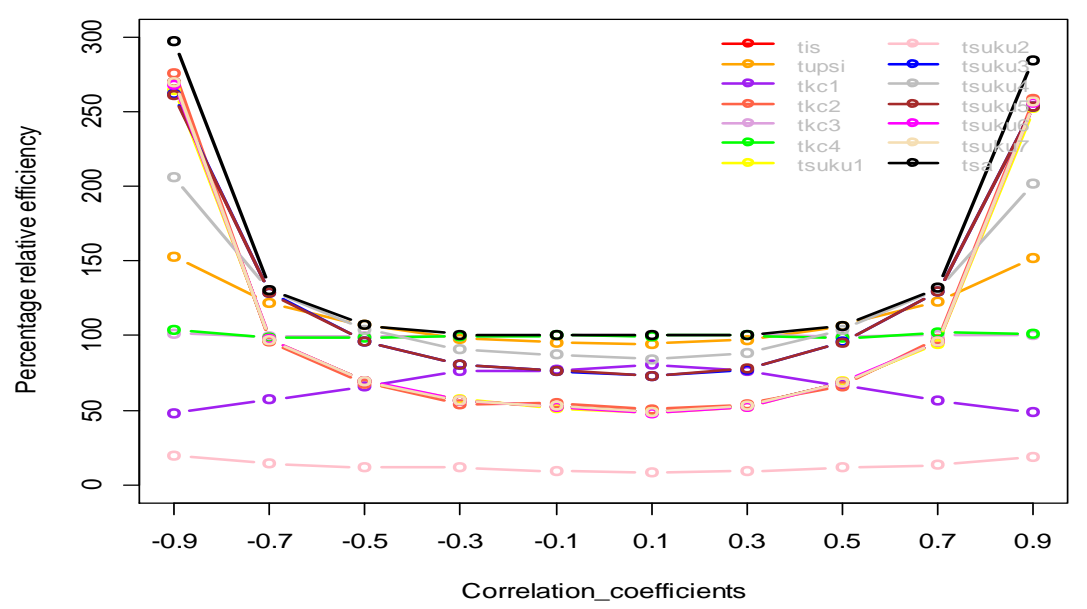

Fig. 1. This shows that the suggested estimator performs better for all the correlation coefficient values (r) over modified ratio-type existing estimators.

\section{Conclusion}

In this paper, we suggested an efficient transformed ratio-type estimator for estimation of population variance of study variable (y) by utilizing single auxiliary information (x) under simple random sampling without replacement. We perceived that our proposed estimator provided Isaki, 1983, a ratio-type estimator, as a particular case of the new estimator $t_{s a}$. The mean squared error and bias of the suggested estimator are derived up-to 1st order approximation. The suggested estimator's performance has been seen through empirical analysis and simulation study, and it can be evaluated that the mean squared error (MSE) and root mean squared error (RMSE) of the suggested estimator is less than the MSE and RMSE of the well-known existing estimators. We proved that our proposed estimator performs better than the competing estimators used in the study as our proposed estimator has a smaller mean squared error over competing estimators. This indicates that our proposed estimator is likely to estimate the variance with less error than other estimators. This also indicates that the confidence intervals for variance based upon our proposed estimator will be more reliable than the confidence intervals constructed by using some other estimator. In addition to this, our proposed estimator is a generalized type estimator and hence can be used in broader situations. Various choices of $\alpha$ will lead to a different estimator, and hence the estimator provides broader applicability compared to the existing estimators. The estimator can be effectively used to estimate variability in different areas of life, e.g., in the medical field, a physician can use it to know the variation in the degree of human blood pressure, body temperature, and heartbeat and pulse rate for an adequate prescription. An agriculturist can use this method to identify all those factors that can affect crop yield. The manufacturer can also use this method to recognize consumers' variation level and disapprove of newly launched products. 


\section{References}

Ahmed, M. S.; Raman, M. S. \& Hossain, M. I. (2000) Some competitive estimators of finite population variance using multivariate auxiliary information. Information and Management Sciences, 11(1):49-54.

Akhlaq, T.; Ismail, M. \& Shahbaz; M. Q. (2019) On efficient estimation of process variability. Journal of symmetry, 11, 554, doi:10.3390/sym11040554.

Chami, P. S.; Sing, B. \& Thomas, D. (2012) A two-parameter ratio-product-ratio estimator using auxiliary information. Journal of Probability and Statistics, article id 103860, doi:10.5402/2012/103860

Gujarati, D. N \& Sangeetha. (2004) Basic econometrics (4th Ed.). New Delhi: TATA McGRAW HILL Companies. Pp. 437.

Isaki, C. T. (1983) Variance estimation using auxiliary information. Journal of the American Statistical Association, 78:117123.

Ismail, M.; Kanwal, N. \& Shahbaz, M.Q. (2018) Generalized ratio-product-type estimator for variance using auxiliary information in simple random sampling. Kuwait Journal of Science, 45(1), 67-75.

Kadilar, C. \& Cingi, H. (2006) Ratio estimators for the population variance in simple and stratified random sampling. Journal of Applied Mathematics and Computation, 173, 1047-1059.

Singh, G. N. \& Suman, S. (2019) An ameliorated two-stage randomized response model for estimating a rare stigmatized characteristic using poisson distribution. Kuwait Journal of Science 46(2), 21-30.
Singh, H. P. \& Solanki, R. S. (2013a) A new procedure for variance estimation in simple random sampling using auxiliary information. Stat Papers, 54: 479-497

Singh, H. P. \& Solanki, R. S. (2013b) Improved estimation of finite population variance using auxiliary information. Communication Statistics-Theory and Methods, 42: 2718-2730.

Subramani, J. \& Kumarapandiyan, G. (2012a) Variance estimation using median of the auxiliary variable. International Journal of Probability and Statistics, 1(3), 62-66.

Subramani, J. \& Kumarapandiyan, G. (2012b) Variance estimation using quartiles and their functions of an auxiliary variable. International Journal of Statistics and Applications, 2(5), 67-72.

Subramani, J. \& Kumarapandiyan, G. (2013) Estimation of variance using known coefficient of variation and median of an auxiliary variable. Journal of Modern Applied Statistical Methods, 12(1), 58-64.

Upadhyaya, L. N. \& Singh, H. P. (1999) An estimator for population variance that utilizes the kurtosis of an auxiliary variable in survey sampling. Vikram Mathemat. Journal, 19: 14-17.

Yadav, R.; Upadhyaya, L. N.; Singh, H. P. \& Chatterjee, S. (2013) A generalized family of transformed ratio-product estimators for variance in sample surveys. Communications in Statistics-Theory and Methods, 42: 1839-1850.

Yadav, S. K. \& Kadilar, C. (2014) A twoparameter variance estimator using auxiliary information. Journal of Applied Mathematics and Computation, 226, 117122. 
Efficient transformed ratio-type estimator using single auxiliary information

$\begin{array}{ll}\text { Submitted } & : 17 / 01 / 2020 \\ \text { Revised } & : 30 / 05 / 2020 \\ \text { Accepted } & : 26 / 07 / 2020 \\ \text { DOI } & : 10.48129 / \text { kjs.v48i2.9030 }\end{array}$

